\title{
Constructions of Strict Left (Right)-Conjunctive Left (Right) Semi-Uninorms on a Complete Lattice
}

\author{
Yuan Wang ${ }^{1, ~ *, ~ K e m i n g ~ T a n g ~}{ }^{1}$, Zhudeng Wang ${ }^{2}$ \\ ${ }^{1}$ College of Information Engineering, Yancheng Teachers University, Yancheng, People's Republic of China \\ ${ }^{2}$ School of Mathematics and Statistics, Yancheng Teachers University, Yancheng, People's Republic of China
}

Email address:

yctuwangyuan@163.com (Yuan Wang),tkmchina@126.com (Keming Tang), zhudengwang2004@163.com (Zhudeng Wang)

*Corresponding author

\section{To cite this article:}

Yuan Wang, Keming Tang, Zhudeng Wang. Constructions of Strict Left (Right)-Conjunctive Left (Right) Semi-Uninorms on a Complete Lattice. International Journal of Intelligent Information Systems. Vol. 6, No. 1, 2017, pp. 1-6. doi: 10.11648/j.ijiis.20170601.11

Received: January 6, 2017; Accepted: January 19, 2017; Published: February 23, 2017

\begin{abstract}
In this paper, we further investigate the constructions of fuzzy connectives on a complete lattice. We firstly illustrate the concepts of strict left (right)-conjunctive left (right) semi-uninorms by means of some examples. Then we give out the formulas for calculating the upper and lower approximation strict left (right)-conjunctive left (right) semi-uninorms of a binary operation.
\end{abstract}

Keywords: Fuzzy Logic, Fuzzy Connective, Left (Right) Semi-Uninorm, Strict Left (Right)-Conjunctive

\section{Introduction}

Uninorms, introduced by Yager and Rybalov [1], and studied by Fodor et al. [2], are special aggregation operators that have proven useful in many fields like fuzzy logic, expert systems, neural networks, aggregation, and fuzzy system modeling (see [3-6]).

This kind of operation is an important generalization of both $t$-norms and $t$-conorms and a special combination of $t$-norms and $t$-conorms (see [2]). But, there are real-life situations when truth functions cannot be associative or commutative. By throwing away the commutativity from the axioms of uninorms, Mas et al. introduced the concepts of left and right uninorms on $[0,1]$ in [7] and later in a finite chain in [8], and Wang and Fang [9-10] studied the left and right uninorms on a complete lattice. By removing the associativity and commutativity from the axioms of uninorms, Liu [11] introduced the concept of semi-uninorms, and $\mathrm{Su}$ et al. [12] discussed the notions of left and right semi-uninorms, on a complete lattice. On the other hand, it is well known that a uninorm (semi-uninorm) $U$ can be conjunctive or disjunctive whenever $U(0,1)=0$ or 1 , respectively. This fact allows us to use uninorms in defining fuzzy implications (see $[9,11$, 13-14]).

Constructing fuzzy connectives is an interesting topic. Recently, Jenei and Montagna [15] introduced several new types of constructions of left-continuous $t$-norms, Wang [16] laid bare the formulas for calculating the smallest pseudo- $t$-norm that is stronger than a binary operation and the largest implication that is weaker than a binary operation, $\mathrm{Su}$ et al. [12] studied the constructions of left and right semi-uninorms on a complete lattice, Su and Wang [17] investigated the constructions of implications and coimplications on a complete lattice. and Wang et al. [18-20] studied the relations among implications, coimplications and left (right) semi-uninorms, on a complete lattice. Moreover, Wang et al. [21-22] investigated the constructions of conjunctive left (right) semi-uninorms, disjunctive left (right) semi-uninorms, strict left (right)-disjunctive left (right) semi-uninorm, implications satisfying the neutrality principle, coimplications satisfying the neutrality principle, and coimplications satisfying the order property.

This paper is a continuation of [12, 21-22]. Motivated by these works in [12, 21-22], we will further focus on this issue and investigate constructions of the upper and lower approximation strict left (right)-conjunctive left (right) semi-uninorms on a complete lattice.

This paper is organized as follows. In Section 2, we recall some necessary concepts about the left (right) semi-uninorms on a complete lattice and illustrate these notions by means of some examples. In Section 3, we give out the formulas for calculating the upper and lower approximation strict left (right)-conjunctive left (right) semi-uninorms of a binary 
operation.

The knowledge about lattices required in this paper can be found in [23].

Throughout this paper, unless otherwise stated, $L$ always represents any given complete lattice with maximal element 1 and minimal element $0 ; J$ stands for any index set.

\section{Strict Conjunctive Left and Right Semi-Uninorms}

Noting that the commutativity and associativity are not desired for aggregation operators in a number of cases, Liu [11] introduced the concept of semi-uninorms and $\mathrm{Su}$ et al. [12] studied the notions of left and right semi-uninorms. Here, we recall some necessary definitions and give some examples of the left and right semi-uninorms on a complete lattice.

Definition 2.1 (Su et al. [12]). A binary operation $U$ on $L$ is called a left (right) semi-uninorm if it satisfies the following two conditions:

(U1) there exists a left (right) neutral element, i.e., an element $e_{L} \in L \quad\left(e_{R} \in L\right)$ satisfying $U\left(e_{L}, x\right)=x$ $\left(U\left(x, e_{R}\right)=x\right.$ for all $x \in L$,

(U2) $U$ is non-decreasing in each variable.

Clearly, $U(0,0)=0$ and $U(1,1)=1$ hold for any left (right) semi-uninorm $U$ on $L$.

If a left (right) semi-uninorm $U$ is associative, then $U$ is the left (right) uninorm [9-10] on $L$.

If a left (right) semi-uninorm $U$ with the left (right) neutral element $e_{L} \in L \quad\left(e_{R} \in L\right)$ has a right (left) neutral element $e_{R} \in L \quad\left(e_{L} \in L\right)$, then $e_{L}=U\left(e_{L}, e_{R}\right)=e_{R}$. Let $e=e_{L}=e_{R}$. Here, $U$ is the semi-uninorm [11].

For any left (right) semi-uninorm $U$ on $L, U$ is said to be left-conjunctive and right-conjunctive if $U(0,1)=0$ and $U(1,0)=0$, respectively. $U$ is said to be conjunctive if both $U(1,0)=0$ and $U(1,0)=0$ since it satisfies the classical boundary conditions of AND.

$U$ is said to be strict left-conjunctive and strict rightconjunctive if $U$ is conjunctive and for any $x \in L, U(x, 1)=0 \Leftrightarrow x=0$ and $U(1, x)=0 \Leftrightarrow x=0$, respectively.

Definition 2.2 (Wang and Fang [9]). A binary operation $U$ on $L$ is called left (right) arbitrary $\vee$-distributive if

$$
\begin{array}{r}
U\left(\vee_{j \in J} x_{j}, y\right)=\vee_{j \in J} U\left(x_{j}, y\right) \quad \forall x_{j}, y \in L \\
\left(U\left(x, \vee_{j \in J} y_{j}\right)=\vee_{j \in J} U\left(x, y_{j}\right) \quad \forall x, y_{j} \in L\right) ;
\end{array}
$$

left (right) arbitrary $\wedge$-distributive if

$$
\begin{array}{r}
U\left(\wedge_{j \in J} x_{j}, y\right)=\wedge_{j \in J} U\left(x_{j}, y\right) \quad \forall x_{j}, y \in L \\
\left(U\left(x, \wedge_{j \in J} y_{j}\right)=\wedge_{j \in J} U\left(x, y_{j}\right) \quad \forall x, y_{j} \in L\right) .
\end{array}
$$

If a binary operation $U$ is left arbitrary $\vee$-distributive $(\wedge$ distributive) and also right arbitrary $\vee$-distributive $(\wedge$ -distributive), then $U$ is said to be arbitrary $\vee$-distributive ( $\wedge$-distributive).

Noting that the least upper bound of the empty set is 0 and the greatest lower bound of the empty set is 1 , we have

$$
\begin{gathered}
U(0, y)=U\left(\vee_{j \in \Phi} x_{j}, y\right)=\vee_{j \in \Phi} U\left(x_{j}, y\right)=0 \\
\left(U(x, 0)=U\left(x, \vee_{j \in \Phi} y_{j}\right)=\vee_{j \in \Phi} U\left(x, y_{j}\right)=0\right)
\end{gathered}
$$

for any $x, y \in L$ when $U$ is left (right) arbitrary $\vee$ -distributive,

$$
\begin{array}{r}
U(1, y)=U\left(\wedge_{j \in \Phi} x_{j}, y\right)=\wedge_{j \in \Phi} U\left(x_{j}, y\right)=1 \\
\left(U(x, 1)=U\left(x, \wedge_{j \in \Phi} y_{j}\right)=\wedge_{j \in \Phi} U\left(x, y_{j}\right)=1\right) .
\end{array}
$$

for any $x, y \in L$ when $U$ is left (right) arbitrary $\wedge$ -distributive.

For the sake of convenience, we introduce the following symbols:

$U_{s}^{e_{L}}(L)$ : the set of all left semi-uninorms with the left neutral element $e_{L}$ on $L$;

$U_{s}^{e_{R}}(L)$ : the set of all right semi-uninorms with the right neutral element $e_{R}$ on $L$;

$U_{c s}^{s e_{L}}(L)$ : the set of all strict left-conjunctive left semi-uninorms with the left neutral element $e_{L}$ on $L$;

$U_{c s}^{e_{R} s}(L)$ : the set of all strict right-conjunctive right semi-uninorms with the right neutral element $e_{R}$ on $L$;

$U_{\mathrm{v} c s}^{s e_{L}}(L)$ : the set of all strict left-conjunctive left arbitrary $\checkmark$-distributive left semi-uninorms with the left neutral element $e_{L}$ on $L$;

$U_{c s v}^{e_{R} s}(L)$ : the set of all strict right-conjunctive right arbitrary $\checkmark$-distributive right semi-uninorms with the right neutral element $e_{R}$ on $L$.

Below, we illustrate these notions by means of several examples.

Example 2.1 (Su et al. [12]). Let $e_{L} \in L$,

$$
U_{s W}^{e_{L}}(x, y)=\left\{\begin{array}{c}
y \text { if } x \geq e_{L}, \\
0 \text { otherwise, }
\end{array} U_{s M}^{e_{L}}(x, y)=\left\{\begin{array}{c}
y \text { if } x \geq e_{L}, \\
1 \text { otherwise }
\end{array}\right.\right.
$$

$$
U_{c s M}^{e_{L}}(x, y)=\left\{\begin{array}{rr}
0 & \text { if } x=0 \text { or } y=0 \\
y & \text { if } 0<x \leq e_{L}, y \neq 0 \\
1 & \text { otherwise }
\end{array}\right.
$$

where $x$ and $y$ are elements of $L$. Then $U_{s W}^{e_{L}}$ and $U_{s M}^{e_{L}}$ are, respectively, the smallest and greatest elements of $U_{s}^{e_{L}}(L)$. By Example 2 and Theorem 8 in [18], we see that 
$U_{c s}^{s e_{L}}(L)$ and $U_{v c s}^{s e_{L}}(L)$ are two join-semilattices with the greatest element $U_{c s M}^{e_{L}}$.

Example 2.2. Let $e_{L} \in L$,

$$
U_{c s W}^{s e_{L}}(x, y)=\left\{\begin{array}{cc}
y & \text { if } x \geq e_{L}, \\
\wedge\{a \in L \mid a \neq 0\} & \text { if } 0<x \text { not } \geq e_{L}, y=1, \\
0 & \text { otherwise. }
\end{array}\right.
$$

When $e_{L} \neq 0$ and $\wedge\{a \in L \mid a \neq 0\} \neq 0$, it is straightforward to verify that $U_{c s W}^{s e_{L}}$ is a strict left-conjunctive left semi-uninorm with the left neutral element $e_{L}$. If $U \in U_{c s}^{s e_{L}}(L)$, then

$$
U(x, y) \geq\left\{\begin{array}{cc}
U\left(e_{L}, y\right)=y & \text { when } x \geq e_{L}, \\
\wedge\{a \in L \mid a \neq 0\} & \text { when } 0<x \text { not } \geq e_{L}, y=1, \\
0 & \text { otherwise, }
\end{array}\right.
$$

i.e., $U \geq U_{c s W}^{s e_{L}}$. Thus, $U_{c s W}^{s e_{L}}$ is the smallest element of $U_{c s}^{s e_{L}}(L)$.

Moreover, assume that $\vee\left\{a \in L \mid a\right.$ not $\left.\geq e_{L}\right\}$ not $\geq e_{L}$. For any $x_{j} \in L(j \in J)$, if $\vee_{j \in J} x_{j} \geq e_{L}$, then there exists $j_{0} \in J$ such that $x_{j_{0}} \geq e_{L}$,

$$
\begin{aligned}
& U_{c s W}^{s e_{L}}\left(\vee_{j \in J} x_{j}, y\right)=y=U_{c s W}^{s e_{L}}\left(x_{j_{0}}, y\right) \\
& =\vee_{j \in J} U_{c s W}^{s e_{L}}\left(x_{j}, y\right) \quad \forall y \in L ;
\end{aligned}
$$

if $0<\vee_{j \in J} x_{j}$ not $\geq e_{L}$, then $x_{j}$ not $\geq e_{L}$ for any $j \in J$ and there exists $j_{0} \in J$ such that $0<x_{j_{0}}$ not $\geq e_{L}$,

$$
\begin{aligned}
& U_{c s W}^{s e_{L}}\left(\vee_{j \in J} x_{j}, 1\right)=\wedge\{a \mid a \neq 0\}=U_{c s W}^{s e_{L}}\left(x_{j_{0}}, 1\right) \\
& =\vee_{j \in J} U_{c s W}^{s e_{L}}\left(x_{j}, 1\right) ; \\
& \quad U_{c s W}^{s e_{L}}\left(\vee_{j \in J} x_{j}, y\right)=0=U_{c s W}^{s e_{L}}\left(x_{j_{0}}, y\right) \\
& =\vee_{j \in J} U_{c s W}^{s e_{L}}\left(x_{j}, y\right) \quad y \neq 1 ;
\end{aligned}
$$

if $\vee_{j \in J} x_{j}=0$, then $x_{j}=0$ for any $j \in J$,

$$
U_{c s W}^{s e_{L}}\left(\vee_{j \in J} x_{j}, y\right)=0=\vee_{j \in J} U_{c s W}^{s e_{L}}\left(x_{j}, y\right) \quad \forall y \in L .
$$

Therefore, $U_{c s W}^{s e_{L}}$ is left arbitrary $\vee$-distributive and the smallest element of $U_{\mathrm{vcs}}^{s e_{L}}(L)$.

Example 2.3. Let $e_{R} \in L$,

$$
U_{s W}^{e_{R}}(x, y)=\left\{\begin{array}{l}
x \text { if } y \geq e_{R}, \\
0 \text { otherwise }
\end{array} \quad U_{s M}^{e_{R}}(x, y)=\left\{\begin{array}{l}
x \text { if } y \geq e_{R}, \\
1 \text { otherwise }
\end{array}\right.\right.
$$

$$
\begin{aligned}
& U_{c s M}^{e_{R}}(x, y)=\left\{\begin{array}{lr}
0 & \text { if } x=0 \text { or } y=0 \\
x & \text { if } 0<y \leq e_{R}, x \neq 0 \\
1 & \text { otherwise }
\end{array}\right. \\
& U_{c s W}^{e_{R} s}(x, y)=\left\{\begin{array}{cc}
x & \text { if } y \geq e_{R}, \\
\wedge\{a \in L \mid a \neq 0\} & \text { if } 0<y \text { not } \geq e_{R}, x=1, \\
0 & \text { otherwise. }
\end{array}\right.
\end{aligned}
$$

where $x$ and $y$ are elements of $L$. By Example 2.6 in [20], we know that $U_{s W}^{e_{R}}$ and $U_{s M}^{e_{R}}$ are, respectively, the smallest and greatest elements of $U_{s}^{e_{R}}(L)$. By Example 3 and Theorem 8 in [18], we see that $U_{c s}^{e_{R} s}(L)$ and $U_{c s \vee}^{e_{R} s}(L)$ are two join-semilattices with the greatest element $U_{c s M}^{e_{R}}$.

Similarly, When $e_{L} \neq 0$ and $\wedge\{a \in L \mid a \neq 0\} \neq 0, U_{c s W}^{e_{R} s}$ is the smallest element of $U_{c s}^{e_{R} s}(L)$. Moreover, if $\vee\left\{a \in L \mid a\right.$ not $\left.\geq e_{R}\right\}$ not $\geq e_{R}$, then $U_{c s W}^{e_{R} s}$ is the smallest element of $U_{c s \vee}^{e_{R} s}(L)$.

\section{Constructing Strict Conjunctive Left and Right Semi-Uninorms}

Constructing aggregation operators is an interesting work. Recently, Jenei and Montagna [15] introduced several new types of constructions of left-continuous $t$-norms, Su et al. [12] studied the constructions of left and right semi-uninorms on a complete lattice, and Wang et al. [21-22] investigated the constructions of conjunctive left (right) semi-uninorms and disjunctive left (right) semi-uninorms on a complete lattice. Now, we continue this work and give out the formulas for calculating the upper and lower approximation strict left (right)-conjunctive left (right) semi-uninorms of a binary operation.

It is easy to verify that $\vee_{j \in J} U_{j} \in U_{c s}^{s e_{L}}(L)$ for any nonempty subset $\left\{U_{j} \mid j \in J\right\}$ of $U_{c s}^{s e_{L}}(L)$. If $e_{L} \neq 0$ and $\wedge\{a \in L \mid a \neq 0\} \neq 0$, then $U_{c s}^{s e_{L}}(L)$ is a complete lattice with the smallest element $U_{c s W}^{s e_{L}}$ and greatest element $U_{c s M}^{e_{L}}$ by Example 2.2. Thus, for a binary operation $A$ on $L$, if there exists $U \in U_{c s}^{s e_{L}}(L)$ such that $A \leq U$, then

$$
\wedge\left\{U \mid A \leq U, U \in U_{c s}^{s e_{L}}(L)\right\}
$$

is the smallest strict left-conjunctive left semi-uninorm that is stronger than $A$ on $L$, we call it the upper approximation strict left-conjunctive left semi-uninorm of $A$ and write as $[A)_{c s}^{s e_{L}}$; if there exists $U \in U_{c s}^{s e_{L}}(L)$ such that $U \leq A$, then

$$
\vee\left\{U \mid U \leq A, U \in U_{c s}^{s e_{L}}(L)\right\}
$$

is the largest strict left-conjunctive left semi-uninorm that is weaker than $A$ on $L$, we call it the lower approximation strict left-conjunctive left semi-uninorm of $A$ and write as $(A]_{c s}^{s e_{L}}$. 
Similarly, we introduce the following symbols:

$[A)_{c s}^{e_{R} s}$ : the upper approximation strict right-conjunctive right semi-uninorm of $A$;

$(A]_{c s}^{e_{R} s}$ : the lower approximation strict right-conjunctive right semi-uninorm of $A$;

$[A)_{\vee c s}^{s e_{L}}:$ the upper approximation strict left-conjunctive left arbitrary $\vee$-distributive left semi-uninorm of $A$;

$(A]_{\vee c s}^{s e_{L}}$ : the lower approximation strict left-conjunctive left arbitrary $\vee$-distributive left semi-uninorm of $A$;

$[A)_{c s \vee}^{e_{R} s}$ : the upper approximation strict right-conjunctive right arbitrary $\vee$-distributive right semi-uninorm of $A$;

$(A]_{c s \vee}^{e_{R} s}$ : the lower approximation strict right-conjunctive right arbitrary $\vee$-distributive right semi-uninorm of $A$.

Definition 3.1 (Su et al. [12]). Let $A$ be a binary operation on L. Define the upper approximation aggregator $A_{u a}$ and the lower approximation aggregator $A_{l a}$ of $A$ as follows:

$$
\begin{array}{ll}
A_{u a}(x, y)=\vee\{A(u, v) \mid u \leq x, v \leq y\} & \forall x, y \in L, \\
A_{l a}(x, y)=\wedge\{A(u, v) \mid u \geq x, v \geq y\} & \forall x, y \in L .
\end{array}
$$

Theorem 3.1 ( $\mathrm{Su}$ et al. [12]). Let $A, B \in L^{L \times L}$. Then the following statements hold:

$$
A_{l a} \leq A \leq A_{u a}
$$

$$
(A \vee B)_{u a}=A_{u a} \vee B_{u a} \text { and }
$$

$$
(A \wedge B)_{l a}=A_{l a} \wedge B_{l a}
$$

$A_{u a}$ and $A_{l a}$ are non-decreasing in its each variable.

If $A$ is non-decreasing in its each variable, then

$$
A_{\text {ua }}=A_{l a}=A \text {. }
$$

Theorem 3.2. Let $A \in L^{L \times L}$.

(1) If $A$ is left (right) arbitrary $\vee$-distributive, then $A_{u a}$ is left (right) arbitrary $\vee$-distributive.

(2) If $A$ is left (right) arbitrary $\wedge$-distributive, then $A_{l a}$ is left (right) arbitrary $\wedge$-distributive.

Proof. We only prove that statement (1) holds.

If $A$ is left arbitrary $\vee$-distributive, then $A$ is non-decreasing in its first variable,

$$
\begin{gathered}
A_{u a}(x, y)=\vee\{A(u, v) \mid u \leq x, v \leq y\} \\
=\vee\{A(x, v) \mid v \leq y\} \quad \forall x, y \in L, \\
A_{u a}\left(\vee_{j \in J} x_{j}, y\right)=\vee\left\{A\left(\vee_{j \in J} x_{j}, v\right) \mid v \leq y\right\} \\
=\vee\left\{\vee_{j \in J} A\left(x_{j}, v\right) \mid v \leq y\right\}=\vee_{j \in J}\left\{\vee A\left(x_{j}, v\right) \mid v \leq y\right\} \\
=\vee_{j \in J} A_{u a}\left(x_{j}, y\right) \quad \forall x_{j}, y \in L(j \in J),
\end{gathered}
$$

i.e., $A_{u a}$ is left arbitrary $\vee$-distributive.

Similarly, we can show that $A_{u a}$ is right arbitrary $\vee$ -distributive when $A$ is right arbitrary $\vee$-distributive.

The theorem is proved.

Below, we give out the formulas for calculating the upper and lower approximation strict left (right)-conjunctive left (right) semi-uninorms of a binary operation.

Theorem 3.3. Suppose that $A \in L^{L \times L}, e_{L} \neq 0$ and $\wedge\{a \in L \mid a \neq 0\} \neq 0$.

(1) If $A \leq U_{c s M}^{e_{L}}$, then $[A]_{c s}^{s e_{L}}=U_{c s W}^{s e_{L}} \vee A_{u a}$;

if $U_{c s W}^{s e_{L}} \leq A$, then $(A]_{c s}^{s e_{L}}=U_{c s M}^{e_{L}} \wedge A_{l a}$.

(2) If $\vee\left\{a \in L \mid a\right.$ not $\left.\geq e_{L}\right\}$ not $\geq e_{L}, \quad A \leq U_{c s M}^{e_{L}}$ and $A$ is left arbitrary $\vee$-distributive, then $[A)_{\vee c s}^{s e_{L}}=U_{c s W}^{s e_{L}} \vee A_{u a}$. Moreover, if $A$ is non-decreasing in its second variable, then $[A)_{\vee c s}^{s e_{L}}=U_{c s W}^{s e_{L}} \vee A$.

Proof. Assume that $e_{L} \neq 0$ and $\wedge\{a \in L \mid a \neq 0\} \neq 0$. Then $U_{c S W}^{s e_{L}}$ and $U_{c s M}^{e_{L}}$ are, respectively, the smallest and greatest elements of $U_{c s}^{s e_{L}}(L)$ by Examples 2.1 and 2.2.

(1) Let $U_{1}=U_{c s W}^{s e_{L}} \vee A_{u a}$. If $A \leq U_{c s M}^{e_{L}}$, then $A \leq U_{1}$, $A_{u a} \leq\left(U_{c s M}^{e_{L}}\right)_{u a}=U_{c s M}^{e_{L}}$. Thus,

$$
U_{c s W}^{e_{L}} \leq U_{1} \leq U_{c s M}^{e_{L}}
$$

It implies that $U_{1}(1,0)=U_{1}(0,1)=0$ and $U_{1}\left(e_{L}, y\right)=y$ for any $y \in L$. If $U_{1}(x, 1)=0$, then $U_{c s W}^{s e_{L}}(x, 1)=0$ and so $x=0$, i.e., $U_{1}$ is strict left-conjunctive. By Theorem 3.1(3) and the monotonicity of $U_{c s W}^{s e_{L}}$, we can see that $U_{1}$ is non-decreasing in its each variable. So, $U_{1} \in U_{c s}^{s_{L}}(L)$. If $A \leq U$ and $U \in U_{c s}^{s e_{L}}(L)$, then $A_{u a} \leq U_{u a}=U$ and $U_{1}=U_{c s W}^{s e_{L}} \vee A_{u a} \leq U$. Therefore,

$$
[A)_{c s}^{s e_{L}}=U_{c s W}^{s e_{L}} \vee A_{u a} .
$$

Let $U_{2}=U_{c s M}^{e_{L}} \wedge A_{l a}$. If $U_{c s W}^{s e_{L}} \leq A$, then, $U_{c s W}^{s e_{L}}=\left(U_{c s W}^{s e_{L}}\right)_{l a} \leq A_{l a}$ and $U_{c s W}^{s e_{L}} \leq U_{2} \leq U_{c s M}^{e_{L}}$. Thus, $U_{2}(1,0)=U_{2}(0,1)=0$ and $U_{2}\left(e_{L}, y\right)=y$ for any $y \in L$ and $U_{2}$ is strict left-conjunctive. By Theorem 3.1(3) and the monotonicity of $U_{c s M}^{e_{L}}$, we know that $U_{2}$ is non-decreasing in its each variable. So, $U_{2} \in U_{c s}^{s e_{L}}(L)$. If $U \leq A$ and $U \in U_{c s}^{s e_{L}}(L)$, then $U=U_{l a} \leq A_{l a}$ and $U \leq U_{c s M}^{e_{L}} \wedge A_{l a}=U_{2}$. Therefore,

$$
(A]_{c S}^{s e_{L}}=U_{c s M}^{e_{L}} \wedge A_{l a} .
$$

(2) When $\vee\left\{a \in L \mid a \operatorname{not} \geq e_{L}\right\}$ not $\geq e_{L}, U_{c s W}^{s e_{L}}$ and $U_{c s M}^{e_{L}}$ are, respectively, the smallest and greatest elements of 
$U_{\vee c s}^{s e_{L}}(L)$ by Examples 2.1 and 2.2. Let $U_{3}=U_{c s W}^{s e_{L}} \vee A_{u a}$. If $A \leq U_{c s M}^{e_{L}}$, then $U_{3} \in U_{c s}^{s e_{L}}(L)$ by statement (1). Noting that $A$ is left arbitrary $\vee$-distributive, we can see that $A_{u a}$ is also left arbitrary $\vee$-distributive by Theorem 3.2(1). Thus, $U_{3}$ is left arbitrary $\vee$-distributive and $U_{3} \in U_{\vee c S}^{s e_{L}}(L)$. By the proof of statement (1), we have that $[A)_{\vee c s}^{s e_{L}}=U_{c s W}^{s e_{L}} \vee A_{u a}$.

Moreover, if $A$ is non-decreasing in its second variable, then $A_{u a}=A$ by Theorem 3.1(4) and so

$$
[A)_{\vee c s}^{s e_{L}}=U_{c s W}^{s e_{L}} \vee A
$$

The theorem is proved.

Similarly, for calculating the upper and lower approximation strict right-conjunctive right semi-uninorms of a binary operation, we have the following theorem.

Theorem 3.4. Suppose that $A \in L^{L \times L}, e_{R} \neq 0$ and $\wedge\{a \in L \mid a \neq 0\} \neq 0$.

(1) If $A \leq U_{c s M}^{e_{R}}$, then $[A)_{c s}^{e_{R} s}=U_{c s W}^{e_{R} s} \vee A_{u a}$; if $U_{c s W}^{e_{R} s} \leq A$, then $(A]_{c S}^{e_{R} s}=U_{c s M}^{e_{R}} \wedge A_{l a}$.

(2) If $\vee\left\{a \in L \mid a\right.$ not $\left.\geq e_{R}\right\}$ not $\geq e_{R}, A \leq U_{c s M}^{e_{R}}$ and $A$ is right arbitrary $\vee$-distributive, then $[A)_{c s \vee}^{e_{R} s}=U_{c s W}^{e_{R} s} \vee A_{u a}$. Moreover, if $A$ is non-decreasing in its first variable, then $[A)_{c s \vee}^{e_{R} s}=U_{c s W}^{e_{R} s} \vee A$.

\section{Conclusions and Future Works}

Constructing fuzzy connectives is an interesting topic. Recently, Su et al. [12] studied the constructions of left and right semi-uninorms, and Wang et al. [17-18, 20, 22] investigated the constructions of implications and coimplications on a complete lattice. In this paper, motivated by these works, we give out the formulas for calculating the upper and lower approximation strict left (right)-conjunctive left (right) semi-uninorms of a binary operation.

In a forthcoming paper, we will further investigate the constructions of left (right) semi-uninorms and coimplications on a complete lattice.

\section{Acknowledgements}

This work is supported by Science Foundation of Yancheng Teachers University (16YCKLQ006), the National Natural Science Foundation of China (61379064) and Jiangsu Provincial Natural Science Foundation of China (BK20161313).

\section{References}

[1] R. R. Yager and A. Rybalov, "Uninorm aggregation operators", Fuzzy Sets and Systems, 80, 111-120, 1996.

[2] J. Fodor, R. R. Yager and A. Rybalov, "Structure of uninorms",
Internat. J. Uncertainly, Fuzziness and Knowledge-Based Systems, 5, 411-427, 1997.

[3] D. Gabbay and G. Metcalfe, "fuzzy logics based on [0,1)-continuous uninorms", Arch. Math. Logic, 46, 425-449, 2007.

[4] A. K. Tsadiras and K. G. Margaritis, "The MYCIN certainty factor handling function as uninorm operator and its use as a threshold function in artificial neurons", Fuzzy Sets and Systems, 93, 263-274, 1998.

[5] R. R. Yager, "Uninorms in fuzzy system modeling", Fuzzy Sets and Systems, 122, 167-175, 2001.

[6] R. R. Yager, "Defending against strategic manipulation in uninorm-based multi-agent decision making", European J. Oper. Res., 141, 217-232, 2002.

[7] M. Mas, M. Monserrat and J. Torrens, "On left and right uninorms", Internat. J. Uncertainly, Fuzziness and Knowledge-Based Systems, 9, 491-507, 2001.

[8] M. Mas, M. Monserrat, and J. Torrens, "On left and right uninorms on a finite chain", Fuzzy Sets and Systems, 146, 3-17, 2004.

[9] Z. D. Wang and J. X. Fang, "Residual operators of left and right uninorms on a complete lattice", Fuzzy Sets and Systems, 160, 22-31, 2009.

[10] Z. D. Wang and J. X. Fang, "Residual coimplicators of left and right uninorms on a complete lattice", Fuzzy Sets and Systems, 160, 2086-2096, 2009.

[11] H. W. Liu, "Semi-uninorm and implications on a complete lattice", Fuzzy Sets and Systems, 191, 72-82, 2012.

[12] Y. Su, Z. D. Wang and K. M. Tang, "Left and right semi-uninorms on a complete lattice", Kybernetika, 49, 948-961, 2013.

[13] B. De Baets and J. Fodor, "Residual operators of uninorms", Soft Computing, 3, 89-100, 1999.

[14] M. Mas, M. Monserrat and J. Torrens, "Two types of implications derived from uninorms", Fuzzy Sets and Systems, 158, 2612-2626, 2007.

[15] S. Jenei and F. Montagna, "A general method for constructing left-continuous $t$-norms", Fuzzy Sets and Systems, 136, 263-282, 2003.

[16] Z. D. Wang, "Generating pseudo-t-norms and implication operators", Fuzzy Sets and Systems, 157, 398-410, 2006.

[17] Y. Su and Z. D. Wang, "Constructing implications and coimplications on a complete lattice", Fuzzy Sets and Systems, 247, 68-80, 2014

[18] X. Y. Hao, M. X. Niu and Z. D. Wang, "The relations between implications and left (right) semi-uninorms on a complete lattice", Internat. J. Uncertainly, Fuzziness and Knowledge-Based Systems, 23, 245-261, 2015.

[19] X. Y. Hao, M. X. Niu, Y. Wang and Z. D. Wang, "Constructing conjunctive left (right) semi-uninorms and implications satisfying the neutrality principle", Journal of Intelligent and Fuzzy Systems, 31, 1819-1829, 2016.

[20] Z. D. Wang, "Left (right) semi-uninorms and coimplications on a complete lattice", Fuzzy Sets and Systems, 287, 227-239, 2016. 
[21] X. Y. Hao, M. X. Niu, Y. Wang and Z. D. Wang, "Constructing conjunctive left (right) semi-uninorms and implications satisfying the neutrality principle", Journal of Intelligent and Fuzzy Systems, 31, 1819-1829, 2016.

[22] Z. D. Wang, M. X. Niu and X. Y. Hao, "Constructions of coimplications and left (right) semi-uninorms on a complete lattice”, Information Sciences, 317, 181-195, 2015.

[23] G. Birkhoff, "Lattice Theory", American Mathematical Society Colloquium Publishers, Providence, 1967. 